\title{
Effects of Illumination Intensity on Ornamental Characteristics of Neoregelia
}

Zhan Shuxia, Yu Shaohua, Yu Xinying, Cao Qunyang, Zhao Zhangjian, Wang Weiyong $\overline{-}$

Research and Development Centre of Flower, Zhejiang Academy of Agricultural Sciences, Hangzhou, 311202

$\square$ Corresponding author Email: zahwwy@163.com

Molecular Plant Breeding, 2019, Vol.10, No.15 doi: $10.5376 / \mathrm{mpb} .2019 .10 .0015$

Received: 27 Aug., 2019

Accepted: 27 Sep., 2019

Published: 16 Oct., 2019

Copyright $\odot 2019$ Zhan et al., This article was first published in Molecular Plant Breeding (2018, 16: 5100-5107) in Chinese, and here was authorized to translate and publish the paper in English under the terms of Creative Commons Attribution License, which permits unrestricted use, distribution, and reproduction in any medium, provided the original work is properly cited.

Preferred citation for this article:

Zhan S.X., Yu S.H., Yu X.Y., Cao Q.Y., Zhao Z.J., Wang W.Y., 2019, Effects of illumination intensity on ornamental characteristics of Neoregelia, Molecular Plant Breeding, 10(15): 116-120 (doi: 10.5376/mpb.2019.10.0015)

\begin{abstract}
Neoregelia has high ornamental value, which is tolerant to shade and drought. In addition, the daily maintenance and management of it is easy and convenient, so it is very popular with consumers. However, illumination intensity has great impact on its blade color. The four seasons in the south of Yangtze River are distinct, and cooling and shading in summer are contradictory, resulting in difficulty in production. This study introduced the morphological characteristics and growth habit of Neoregelia. Through a series of experiments in both plain and mountain sites, we confirmed that the optimum illumination intensity for most varieties of Neoregelia was 50 55 klx. During production procedure, 50\% shading rate could ensure illumination intensity under $55 \mathrm{klx}$, but some green blade varieties need weaker illumination intensity. The shading rate should be kept under $70 \%$ to maintain regular blade color. $70 \mathrm{klx}$ illumination intensity could burn the blade of Neoregelia. This study could provide guidance for production and daily management of Neoregelia.
\end{abstract}

Keywords Neoregelia, Illumination intensity, Cultivation technique

Neoregelia is the generic name of Neoregelia plants in Bromeliaceae. It is also called N.spectabilis, Lip Neoregelia, Cup Neoregelia, Carmine Neoregelia, Gai Neoregelia, Red-backed Neoregelia and so on (Huang et al., 2000; Hu, 2003). Originated in tropical rain forest of Central and South America, it is a perennial epiphytic herb with blade leathery banding, compact lotus-like arrangement, colorful, and a water cup formed in the center of the blade cluster for water storage. There are more than 30 native species of this genus, mainly distributed in Brazil, with thousands of commercial species (Hu, 2003; Wang et al., 2017). Neoregelia mostly has medium or small plant type, plant height $5-20 \mathrm{~cm}$, crown breadth $8-50 \mathrm{~cm}$; There are various kinds of spots on the blades, some having longitudinal stripes, and the center of mature plant blades will become red, purple and other different colors, and some varieties have bright spots on the tip of the blades; Florets are blue, white or pink, clustered in the center of the blade clusters. Neoregelia introduced and cultivated in China in the past mainly include Neoregelia spactabilis (medium-sized plant, gray-green Blade surface, purple-red cross-stripes on the back of blades, red spots at the tip of blades about $1 \mathrm{~cm}$, spines at the edge of blades), Meixin Neoregelia (medium-sized plant, green Blade, bronze patches in strong light, reddish in the center of Blade clusters before and after flowering, spines at the edge of blades), Seven-color Neoregelia (medium-sized plant, green blade, many yellow-white longitudinal stripes in the center, the base of the blades turning red before and after flowering, thorns at the edge of the blades), Neoregelia carolinae cv .Flandria (medium-sized plants, olive green blades, yellow-white stripes on the margin of Blade, thorns on the margin of Blade) and so on. Later, many varieties with spots on the Blade surface and some varieties without thorns on the blade margin were introduced. Some varieties with medium-large plant types and many small or even less than $10 \mathrm{~cm}$ in the crown diameter were also introduced, which enriched the variety types of Neoregelia. Neoregelia is better than other ornamental Neoregelia in drought and shade tolerance, cold tolerance, and can absorb formaldehyde, benzene and other toxic substances (Liang, 2009; Liang et al., 2013). It is deeply loved by people. It is not only a good material for interior decoration, but also can be assembled into a certain shape or a single pot for ornamental. It is also often used in the plant landscaping of hotels, shopping malls and other large places, as well as three-dimensional landscaping of ecological pot and plant walls, and has become a 
fashionable flower in the market. Although Neoregelia has strong shade resistance, it needs strong light to make its ornamental best in the production of finished products. The climate of our province is distinct in four seasons. During the high-temperature period in summer, shading measures are often used to cool down. It makes the Neoregelia mostly have longer blades, less clear blade stripes, and greatly reduce the ornamental. There have been many studies on cultivation techniques and seedling propagation of Neoregelia (Xu et al., 2003; Liu, 2005, Chinese flower horticulture, 6:12-16; Yu and Wang, 2011, Chinese forest by-products, 112(3): 31-32; Wang et al., 2016; Li et al., 2016). In order to improve the quality of Neoregelia, we studied the effect of different shading degrees on the ornamental characteristics of Neoregelia, further to provide reference for producers.

\section{Results and Analysis}

\subsection{Scorch}

Neoregelia is a shade-tolerate plant, which cannot adapt to the hot sun. Excessive light will scorch the blades of the Neoregelia and lose its ornamental value. The experimental plain pilot single-layer film mulching (treatment A1, equivalent to $75 \%$ of the outdoor light intensity, the highest light intensity is about 75 klx) N118, N092 upper blades showed obvious scorch, N098 upper blades also had scorch, but the degree was not as serious as the above two varieties, N139 had slight scorch; Other treatments did not have scorching symptoms. There was no scorching symptom in the three experimental treatments in mountainous areas. Only the observation area (covered with only one film, the light intensity was equivalent to $80 \%$ of the outdoor, and the maximum light intensity was about 88 $\mathrm{klx}$ ) had severe scorch.

\subsection{Blade Color}

Blade color and stripes directly affect the ornamental quality of Neoregelia. The blade color of each treatment in the experimental plain (Figure 1) was A4, A3, A2 and A1 from left to right, respectively. Two spotted blade varieties N098 and N118 treated A2 (two-layer film mulching, equivalent to 50\% of the outdoor light intensity, the highest light intensity is about $50 \mathrm{klx}$ ) were the best, showing normal blade color, obvious Blade stripes and better ornamental; The blade color and stripes in A1 treatment were obvious, but the scorch affected ornamental; In treatment A4 (single-layer film plus two-layer shading net mulching, equivalent to $20 \%$ of the outdoor light intensity, the highest Light intensity is about $20 \mathrm{klx}$ ) and treatment A3 (two-layers film plus one-layer shading net mulching, equivalent to $30 \%$ of the outdoor light intensity, the highest light intensity is about $30 \mathrm{klx}$ ) show that the blades are green and the stripes are blurred, which is the manifestation of insufficient light. The blade color of green blade variety N092 and stripe Blade variety N139 show yellow when treated with A1 and A2, which are the manifestation of excessive illumination, while the green blades when treated with A3 and A4, which had better ornamentality.

The blade color of each treatment in mountainous area pilot (figure 2), above is the spotted blade variety N118, below is the striped blade variety $\mathrm{N} 139$, from left to right is treatment B1, B2, B3, respectively. The blade color of N118 treated with B1 (a layer of shading net and a layer of film covering, equivalent to 50\% of the outdoor light intensity, the highest light intensity is about $55 \mathrm{klx}$ ) was the best, mainly purple-red with clear markings, while

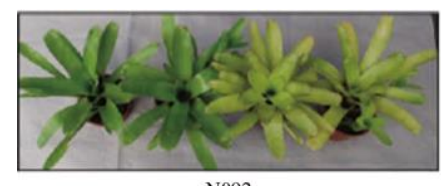

N092

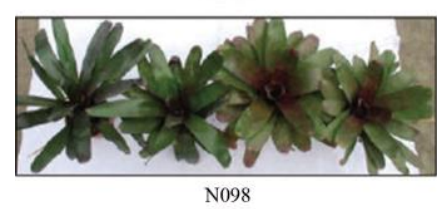

Figure 1 Blade colour of different shading treatments in plain sites
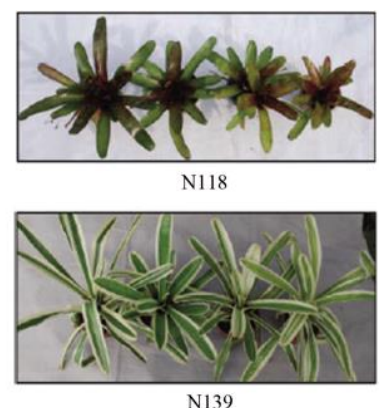

N139 


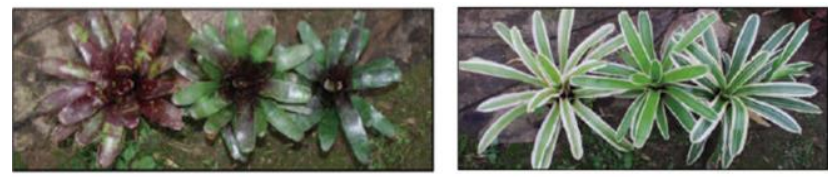

Figure 2 Blade colour of different shading treatments in mountain sites

those of the other two treatments were mainly green with blurred markings. N098 also behaves like N118 (not shown in the figure). The blade color of striped blade variety N139 treated with B1 was yellowing, but its ornamental value was not as good as that of the other two treatments. This phenomenon was also found in all-green blade variety N092 (not shown in the figure).

The above results showed that when the speckled Neoregelia cultivars were shaded by $50 \%$, the speckles were the clearest and the blades were the most beautiful. The blades of green and striped cultivars yellowed when shading was $50 \%$, but their ornamentality became worse. The blades color of green and striped cultivars were better when shading was $70 \% \sim 80 \%$.

\subsection{New Blade Number and Blade Size}

Neoregelia has many blades, which are arranged neatly. The wide and short blades mean good ornamental characteristics. According to the growth circumstance of plants in the experimental plain site, A1 and A2 treatments with strong illumination were better, the average crown width was smaller, more new blades emerged, and the blades were wider and shorter. The best treatment was A2 (two-layer film mulching), that is, $50 \%$ shading (the maximum light intensity was about $50 \mathrm{klx}$ ), with the largest number of newly emerged blades, which was significantly more than A4 treatment; the shortest blade length, which was significantly different from A3 and A4 treatment; and the widest blade was also significantly larger than A4 treatment (Table 1).

The experimental results in mountainous areas were similar, and the difference between treatments was greater. The new blades of treatment B1 (one layer of shading net and one layer of film covering, equivalent to 50\% of outdoor light intensity, and the maximum light intensity was about $55 \mathrm{klx}$ ) were the most and the blades were the shortest, which were significantly different from those of the other two treatments (Figure 3).

The blade of Neoregelia is mainly ornamental. The plant with compact plant type, wide and short blades arranged neatly, bright blades and obvious markings has good ornamental characteristic. The light intensity in cultivation is

Table 1 Growth status of different shading treatments in plain sites

\begin{tabular}{lllll}
\hline Treatment & Crown width $(\mathrm{cm})$ & New blade & Blade length $(\mathrm{cm})$ & Blade width $(\mathrm{cm})$ \\
\hline A1 & $35.5 \mathrm{bC}$ & $6.3 \mathrm{abAB}$ & $28.3 \mathrm{cB}$ & $4.3 \mathrm{aA}$ \\
A2 & $36.4 \mathrm{bBC}$ & $6.7 \mathrm{aA}$ & $27.4 \mathrm{cB}$ & $4.3 \mathrm{aA}$ \\
A3 & $40.0 \mathrm{aAB}$ & $5.8 \mathrm{abAB}$ & $32.7 \mathrm{bA}$ & $4.2 \mathrm{abAB}$ \\
A4 & $42.1 \mathrm{aA}$ & $5.4 \mathrm{bB}$ & $35.5 \mathrm{aA}$ & $3.9 \mathrm{bB}$ \\
\hline
\end{tabular}

Note: Letters behind numbers represented difference between treatments, lowercase letters indicated significant difference $(\mathrm{p}<0.05)$, capital letters indicated extremely significant difference $(\mathrm{p}<0.01)$

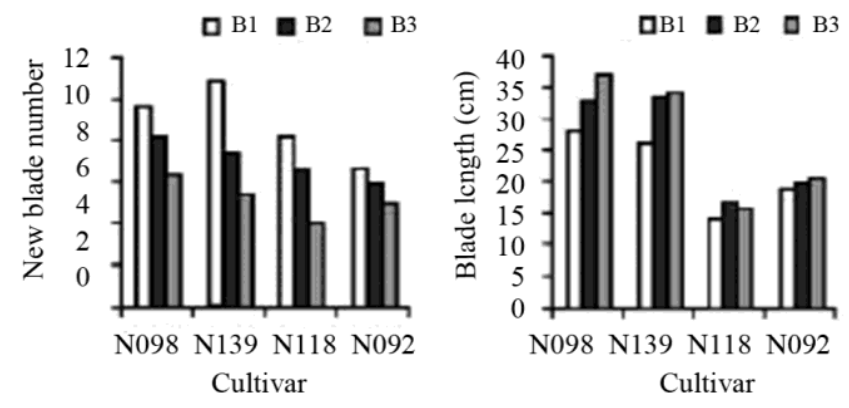

Figure 3 New blade number and blade length in mountain experimental sites 
closely related to the quality of products. The results of this study showed that $50 \%$ shading was the best way to cultivate Neoregelia in Hangzhou area (except for a few green and striped varieties). Most varieties, especially some spotted varieties, could fully display their inherent color and achieve the best ornamental status under this light intensity.

According to the measurement, the highest light intensity in summer is about $100 \mathrm{klx}$ in plain area, $110 \mathrm{klx}$ in mountain area. According to the shading rate of 50\%, the best light intensity in Neoregelia cultivation is 50-55 klx. However, the green blade varieties and some striped blade varieties (such as N092, N139 in this experiment) show a slightly lighter color and a slightly worse gloss. The best light intensity should be about $30 \mathrm{klx}$ (shading rate is about $70 \%)$.

The $25 \%$ shading rate (the strongest illumination exceeded $70 \mathrm{klx}$ ) of the plain experiment showed obvious burns, while the $20 \%$ shading rate (the strongest illumination exceeded $80 \mathrm{klx}$ ) of the mountain experiment showed severe burns, which indicated that the strongest light that the Neoregelia could endure was below $70 \mathrm{klx}$. In addition, the occurrence of blade burns is also related to temperature, water on the blade surface and the tenderness of the plant. Therefore, in order to ensure safety in production, 50\% shading rate is the best choice.

\section{Results}

Summer illumination and temperature are a pair of contradictions that are difficult to coordinate. In plain areas, because of the high temperature, in order to cool down, the sun often overshaded, because the high temperature will also make the blades of Neoregelia too long. It may be feasible to use two layers of net with low shading rate to control separately. Covering two layers at noon and one layer in the morning and evening, in a word, making the light intensity reach or close to $50 \mathrm{klx}$ on the premise of not exceeding the standard temperature can be beneficial to the production of high-quality Neoregelia products.

$\mathrm{Hu}$ et al. (2004) considered that the cultivation of Neoregelia needed adequate sunshine except $30 \%-40 \%$ shade under strong sunshine conditions in summer. Liang (2009) believed that the production of Neoregelia in Xiamen should be maintained at 30\%-50\% illumination level throughout the year. The results of this study are different from those of the above two studies. " $30 \%$ shading in summer" in Hangzhou area has the risk of burning, and the risk of non-shading in autumn is greater. According to our previous measurements, the strongest illumination intensity can reach 70-80 klx before and after National Day without covering the shade net in plastic greenhouse, which is enough to burn the upper blades of Neoregelia. However, "maintaining 30-50\% illumination level throughout the year" seems too conservative. The illumination in late autumn, early spring and winter is not strong and can be non-shaded. The reason of different conclusions may be related to the different test sites and the local air pollution situation.

Studies on grapes by Xing and Pan (2016) suggested that shading weakened the activity of Vv5GT3 promoter related to anthocyanin 3,5-O-diglucoside synthesis. Whether it is the same on Neoregelia remains to be studied in molecular biology.

\section{Materials and Methods}

\subsection{Varieties for Test}

Four cultivars were tested, N092: small and medium-sized plants, green blade type, non-thorny margin, before and after flowering, the center of the blade cluster turns red with obvious red-green boundary; N098: medium-large plants, spotted blade type, purple-red blades, with many round or oval green spots of varying sizes in the middle, with thorns on the margin; N118: small plants, spotted blade type, purple-red blades, with many round or oval green spots of varying sizes in the middle, with thorns on the margin; N139: Medium-large plant type, striped blade type, olive green blades, with a slightly wide yellow-white longitudinal band on the edge, some plants have one or several yellow-white thin longitudinal strips in the middle of the blades, without spines on the margin. N118 is $12 \mathrm{~cm}$ potted seedling, N09 2 and N139 are $14 \mathrm{~cm}$ potted seedling and N098 is $16 \mathrm{~cm}$ potted 
seedling.

\subsection{Test sites and Treatment Methods}

The experiment began on 20 May 2016 and ended on 25 September 2016. The management measures such as fertilization and watering are in accordance with the actual situation of each pilot project, and the management of each major section is consistent. The experiment was carried out in plain and mountainous areas. The experiment in plain site was carried out in the experimental greenhouse of the Flower Research and Development Center of Zhejiang Academy of Agricultural Sciences. Four treatments were set up: single-layer film covering (treatment A1), two-layer film covering (treatment A2), two-layer film plus one-layer sun-shading net covering (treatment A3), single-layer film plus two-layer sun-shading net covering (treatment A4). 8/4 measured the light intensity, the light intensity of each treatment was $75 \%, 50 \%, 30 \%, 20 \%$ of the outdoor respectively. Each treatment area has a 3-meters wide protected area, each treatment area has 40 basins for each variety. Pilot projects in mountainous areas were conducted in Lin Iiatang Village, Linan, at an altitude of $800 \mathrm{~m}$. One-layer shading net plus one-layer film covering (treatment B1), two-layers shading net plus one-layer film covering (treatment B2), and three-layers shading net plus one-layer film covering (treatment B3) were set up. 8/3 measured the light intensity, the light intensity of each treatment is equivalent to $50 \%, 28 \%, 15 \%$ of the outdoor. Each treatment area has a $3 \mathrm{~m}$ wide protected area, each area has 40 basins for each variety. In addition, an observation area covered with only one-layer film is set up. The light intensity is equivalent to $80 \%$ of the outdoor light intensity. 10 basins per variety are used to observe the degree of burns.

\section{Authors' Contributions}

Zhan Shuxia is the experimental designer and the executor of the experimental research, completing the data analysis and the writing of the first draft of the paper; Yu Xinying, Yu Shaohua, Cao Qunyang and Zhao Zhangjian participate in the experimental investigation records; W Wang Weiyong is the conceiver of the project and participates in the experimental design and data analysis. All the authors read and agree to the final text.

\section{Acknowledgments}

This study was funded by the Natural Science Foundation of China (31700336) and Hangzhou Seed and Seedling Project (20150932H14).

\section{References}

Hu J.Y., Xia G.L., and Liang J., 2004, Cultivation techniques ofNeoregelia, Tianjin Nongye Kexue (Tianjin Agricultural Sciences), 10(3): 30-31

Hu S.H., ed., 2003, Bromeliaceae, China Forestry Press, Beijing,China, pp.154-171

Huang Z.M., Zhang Y.L., and Zhong Z.Q., eds., 2000, Bromeliaceae,Guangdong Science and Technology Press, Guangzhou,China, pp.39-41

Li C.H., Li T.C., and Li K.C., 2016, Neoregelia breeding andgreenhouse production, Zhongguo Huahui Yuanyi (China Flowers \& Horticulture), (22): 26-29

Liang S., Shen H.Y., Chen X.H., and Lin L.Q., 2013, Comparativestudy of the absorbility of indoor decorative plant forformaldehyde and benzene, Anquan Yu Huanjing Xuebao(Journal of Safety and Environment), 13(1): 57-62

Liang Y.Q., 2009, Growth habit and management methods of Neoregelia,Yaredai Zhiwu Kexue (Subtropical Plant Science),38(2): 54-56

Wang W.Y., Yu S.H., Zhan S.X., Yu X.Y., Zhao Z.J., and Zhu Q.,2017, Evaluation of ornamental characteristics of Neoreglia germplasm resources, Fenzi Zhiwu Yuzhong (Molecular Plant Breeding), 15(4): 1506-1514

Wang Y.Z., Zhao J., Li L. Kong C.C., and Wang J.P., 2016, Studyon induction of Neoregelia carolinae callus and adventitious bud differentiation, Shanxi Nongye Daxue Xuebao (Journalof Shanxi Agriculture University), 36(2): 91-95

Xing R.R., and Pan Q.H., 2016, Molecular cloning and light response of the Vv5GT3 gene pomoter from cabernet sauvignon (Vitis vinifera L.), Jiyinzuxue Yu Yingyong Shengwuxue(Genomics and Applied Biology), 35(11): 3128-3134

Xu L., Li Z.Y., Lai Q.X., and Li K.L., 2003, Tissue culture andrapid propagation of Neoregelia carolinae, Zhiwu Shenglixue Tongxun (Plant Physiology Communications), 39(4): 344 